\title{
Enrolment criteria for diabetes cardiovascular outcome trials do not inform on generalizability to clinical practice: The case of glucagon-like peptide-1 receptor agonists
}

\author{
Veronica Sciannameo $^{1}$ | Paola Berchialla ${ }^{2}$ | Emanuela Orsi ${ }^{3}$ | Olga Lamacchia ${ }^{4}$ | \\ Susanna Morano $^{5}$ | Fabrizio Querci ${ }^{6}$ | Agostino Consoli ${ }^{7}$ | Angelo Avogaro ${ }^{8}$ (c) | \\ Gian Paolo Fadini ${ }^{-1}$ on behalf of the DARWIN-T2D study
}

${ }^{1}$ Unit of Biostatistics, Epidemiology and Public Health, Department of Cardiac, Thoracic, Vascular Sciences and Public Health, University of Padova, Padova, Italy

${ }^{2}$ Department of Clinical and Biological Sciences, University of Turin, Turin, Italy

${ }^{3}$ Unit of Endocrinology and Metabolic Diseases, Fondazione IRCCS Ca' GrandaOspedale Maggiore Policlinico di Milano, Milan, Italy

${ }^{4}$ Unit of Endocrinology, Department of Medical and Surgical Sciences, University Hospital of Foggia, Foggia, Italy

${ }^{5}$ Unit of Diabetes Complications, V Clinica Medica, Department of Experimental Medicine, University of Rome "La Sapienza", Rome, Italy

${ }^{6}$ Unit of Diabetology, ASST Bergamo Est, Alzano Lombardo, Italy

${ }^{7}$ Department of Medicine and Aging Science, "G. D'Annunzio" University of Chieti, Chieti, Italy

${ }^{8}$ Department of Medicine, University of Padova, Padova, Italy

Correspondence

Gian Paolo Fadini, MD, Associate Professor of Endocrinology, Department of Medicine, University of Padova, Via Giustiniani 2, 35128 Padova, Italy.

Email: gianpaolofadini@hotmail.com

Funding information

This study was supported by the Italian Diabetes Society and the University of Padova.

Peer Review

The peer review history for this article is available at https://publons.com/publon/10. 1111/dom.13962.

\begin{abstract}
Aim: To evaluate the generalizability of cardiovascular outcome trials (CVOTs) on glucagon-like peptide-1 receptor agonists (GLP-1RAs), we assessed what proportion of real-world patients with type 2 diabetes (T2D) constitute true CVOT-like populations.
\end{abstract}

Materials and Methods: We applied inclusion/exclusion (I/E) criteria of each GLP1RA CVOT to a cross-sectional database of 281380 T2D patients from Italian diabetes outpatient clinics. We calculated the proportion of patients eligible for each CVOT and compared their clinical characteristics with those of trial patients. In addition, we used a Bayesian network-based method to sample the greatest subsets of real-world patients yielding true CVOT-like populations.

Results: Between 98725 and 124164 T2D patients could be evaluated for CVOT eligibility. After excluding patients who were already on GLP-1RAs and applying I/E criteria, $35.8 \%$ of patients would be eligible for REWIND, 34.1\% for PIONEER-6, 13.4\% for EXSCEL, $10.1 \%$ for SUSTAIN-6, $9.5 \%$ for HARMONY and $9.4 \%$ for LEADER. Overall, $45.4 \%$ of patients could be eligible for at least one of the CVOTs. These patients, however, were extremely different to trial patients in most of the clinical characteristics, including demographics, concomitant medications and complications. The greatest CVOT-like subsets of real-world patients were $0.5 \%$ for SUSTAIN-6, 1.0\% for EXSCEL, 1.2\% for LEADER, 1.8\% for PIONEER-6 and $7.9 \%$ for REWIND.

Conclusions: A very small proportion of real-world patients constitute true CVOTlike populations. These findings question whether any meaningful information can be drawn from applying trial enrolment criteria to real-world T2D patients.

\section{KEYWORDS}

cardiovascular disease, glucagon-like peptide-1 analogue, pharmaco-epidemiology, population study, type 2 diabetes 


\section{1 | INTRODUCTION}

In the field of diabetes pharmacotherapy, cardiovascular outcome trials (CVOTs) have been designed to test the cardiovascular safety of glucose-lowering medications (GLMs) against placebo. ${ }^{1}$ Although designed primarily to show non-inferiority, some CVOTs have shown that active treatment was superior to placebo in reducing the rates of major adverse cardiovascular outcome events (MACE) in patients with type 2 diabetes (T2D). Results of these CVOTs have been incorporated into consensus algorithms, which now prioritize certain GLMs for the prevention of cardiovascular complications. $^{2}$

This is the case for glucagon-like peptide-1 receptor agonists (GLP-1RAs) and sodium-glucose co-transporter-2 inhibitors (SGLT-2is). Among GLP-1RAs, dedicated CVOTs showed the superiority of liraglutide (LEADER), semaglutide (SUSTAIN-6), albiglutide (HARMONY) and dulaglutide (REWIND) versus placebo in reducing the rates of three-point MACE (cardiovascular death, non-fatal myocardial infarction or stroke). ${ }^{3-6}$ The CVOTs on exenatide once-weekly (exeOW) and oral semaglutide showed nominal reductions in mortality rates. ${ }^{7,8}$ Therefore, there is general agreement that GLP-1RAs as a class improve the cardiovascular outcomes of T2D. ${ }^{9}$

However, the generalizability of CVOT findings to clinical practice may be hampered by the many differences between trial settings and routine care. CVOTs recruit patients based on rigorous inclusion/exclusion (I/E) criteria and closely follow them at regular intervals within strict trial experimental protocols. CVOTs are event-driven, and their I/E criteria allow rapid collection of the desired number of events by selecting T2D patients with a prior history of cardiovascular disease and/or multiple risk factors. Because only $\sim 30 \%$ of T2D patients in routine care show signs of macroangiopathy, to what extent the results of CVOTs can be translated to T2D patients at lower cardiovascular risk is a matter of debate. Positive results from REWIND, in which $\sim 30 \%$ of patients had established cardiovascular disease, ${ }^{3}$ suggest that the benefit of GLP-1RAs may be independent from the baseline cardiovascular risk. ${ }^{9,10}$

A few studies have examined what proportion of T2D patients from various clinical care settings would satisfy I/E criteria to be enrolled in specific CVOTs, ${ }^{11-14}$ but no study to date has examined what the proportion is of real-world patients that corresponds to CVOT populations. Indeed, applying CVOT enrolment criteria to real-world T2D patients yields subgroups that are substantially different from the actual CVOT populations, thereby leaving the question of generalizability unanswered.

In this study, we aimed to resolve this issue. After applying I/E criteria for GLP-1RA CVOTs to a T2D real-world population, we show by how much the eligible population of patients differs from those of CVOTs, and calculate what proportion of patients from routine care would generate a true CVOT-like population.

\section{2 | METHODS}

\subsection{Data source}

The DARWIN-T2D (DAta for Real World evldeNce in Type 2 Diabetes) study was conducted by the Italian Diabetes Society and was initially designed to evaluate dapagliflozin in the real world. Protocol details and primary analysis have been reported previously. ${ }^{15,16}$ The DARWIN-T2D database contains cross-sectional information on 281000 T2D patients from 46 diabetes outpatient clinics across Italy. Data were collected at each centre at the last available appointment attended by each patient in 2015 and 2016. Each clinic used the same electronic chart system to store patients' data (MyStar Connect/ Smart Digital Clinic, Meteda Srl, San Benedetto del Tronto, Italy). The relevant data were extracted by dedicated software (Meteda) without manual intervention.

We recorded the following information: demographics (age, sex, diabetes duration), anthropometrics (height, weight, body mass index [BMI], waist circumference), cardiovascular risk factors (smoking, blood pressure values, lipid profile), estimated glomerular filtration rate (Chronic Kidney Disease Epidemiology Collaboration Equation ${ }^{17}$ ) and other laboratory data (including urinary albumin excretion rate and liver enzymes), and medications for the treatment of diabetes and other cardiovascular risk factors or conditions. In addition, detailed information was collected on diabetic complications from ICD-9 codes in the electronic chart, including: presence and stage of retinopathy and diabetic macular oedema; presence or absence of somatic or autonomic diabetic neuropathy; history of stroke, transient ischaemic attack or carotid endoarterectomy/stenting; history of angina, myocardial infarction or coronary revascularization; presence/absence of left ventricular hypertrophy and history of heart failure; history of claudication, limb ischaemia or amputation; presence of asymptomatic atherosclerosis of coronary, carotid or leg arteries. Codes to define prevalent cardiovascular disease have been standardized in the electronic chart, which is routinely used to monitor the quality of diabetes care in Italy. ${ }^{18}$

Not all of the records were complete for all of the patients: the degree and distribution of missing variables have been reported previously. ${ }^{15}$

\subsection{Data analysis}

From the respective publications, we retrieved I/E criteria regarding the following CVOTs on GLP-1RAs: LEADER (liraglutide), ${ }^{6}$ SUSTAIN-6 (semaglutide), ${ }^{5}$ EXSCEL (exeOW), ${ }^{7}$ REWIND (dulaglutide), ${ }^{3}$ PIONEER6 (oral semaglutide) ${ }^{8}$ and HARMONY (albglutide). ${ }^{4}$ Details of these trials were published during 2015-2019, a timeframe which partially overlaps with our data collection period (2015-2016). Specific I/E criteria had to be adapted to the available data in the DARWIN-T2D database with some modifications (Table S1). For instance, the following information used for CVOT I/E criteria was not available: myocardial ischaemia stress test or imaging; diastolic dysfunction; 
ankle-brachial index; calcitonin levels; and history of cancer and pancreatitis. The timings of prior cardiovascular events and revascularization were not available so as to exclude patients with recent events. Because no information concerning contraception was available, women of childbearing potential were excluded. Patients with missing information for key I/E variables were also excluded from the analysis.

We therefore identified T2D patients who would be eligible to participate in each of the considered CVOTs and calculated the respective proportion over the total background population of patients with available data. Then we compared the average clinical characteristics of eligible patients with average clinical characteristics of patients in the database who were already on GLP-1RAs, and also with those of patients actually enrolled in CVOTs (from the respective publications).

Finally, from the DARWIN-T2D database, we extracted the largest subgroup of patients who had average clinical characteristics superimposable on those of CVOTs. Because no specific tool was already available for this case-sampling procedure, we devised an analytical strategy, which is described below.

\section{3 | Statistical analysis}

For descriptive purposes, continuous variables are expressed as means and standard deviations, whereas categorical variables are expressed as percentages. To evaluate to what extent two groups of patients were similar, we computed the absolute standardized mean difference (SMD) for each variable. Conventionally, an SMD value of 0.1 or less is considered indicative of a good balance. For example, for continuous variables, an SMD of $<0.1$ indicates that the difference between the means of the two groups is $<10 \%$ of the pooled standard deviation. Because of the very large sample size in each comparison, $P$-values were not calculated, as several minor and clinically irrelevant differences would yield $P$-values of $<0.05$.

\section{4 | Sampling CVOT-like populations}

Continuous variables in the DARWIN-T2D database were categorized into five classes, on the basis of each trial's summary statistics, and assuming a normal distribution. Observations with at least one missing value were removed. A Bayesian network $(B N)$ was constructed for each randomized controlled trial (RCT), because each variable was preprocessed (continuous variables were categorized) according to the specific RCT's summary statistics, on the case complete dataset to obtain the conditional probability distributions, which reflect the dependencies among variables. All of the available variables were included in the construction of the BN. Then the Peter-Clark stable algorithm with a 100 -fold bootstrap was employed for the structural learning of the $\mathrm{BN}$ (ie, for identifying the relationships among variables). ${ }^{19}$ A more robust $\mathrm{BN}$ was obtained by averaging the $100 \mathrm{BNs}$ learned, considering only the relationships among variables present in at least $95 \%$ of times. ${ }^{20}$ Bootstrap was performed to address the sample variability. Finally, a maximum a posteriori estimation was used to compute the set of probabilities for conditional nodes, whereas for unconditional nodes, probabilities were assigned computing the ratio between CVOT frequency and DARWIN-T2D frequency, for each variable category, and then normalizing to 1 . This was carried out to take into account the different distribution of patients' characteristics between DARWIN-T2D and CVOTs, and to increase the probability of the patients selected being less represented in DARWIN-T2D than CVOTs, and to decrease the probability of the patients selected being more represented in DARWIN-T2D than CVOTs. The decomposition of the joint probability into the product of conditional and unconditional probabilities was used to achieve a final probability of inclusion in a CVOT for each patient in DARWIN-T2D. ${ }^{21}$ The balancing between the patients sampled and each trial's patients was evaluated through SMD. To achieve an SMD smaller than 0.1 we proceeded as follows: first, we balanced the two groups according to an SMD smaller than 0.2 for each variable. To obtain this result, for each variable with an SMD greater than $0.2,2 \%$ of patients with values in the tails of the distribution were sampled and removed from the DARWIN-T2D dataset. This procedure was iterated until SMD was less than 0.2 .

The entire procedure was repeated to achieve a new random sample of patients balanced according to an SMD smaller than 0.2. Finally, all of the balanced groups obtained were joined together, and, for each variable, to once again obtain an SMD smaller than 0.1, the same procedure was applied. A sensitivity analysis on different thresholds of SMD was carried out, and the choice of the double threshold 0.2 and 0.1 produced the best balanced group. All of the analyses were performed using $\mathrm{R}$ version 3.5.0 ( $\mathrm{R}$ Development Core Team).

\section{3 | RESULTS}

The original dataset was composed of 281380 patients. Because the minimum requirement to enter the database was T2D diagnosis, many patients had missing values for several of the variables needed to evaluate CVOT I/E criteria. Among 130380 patients with available information on GLMs, 6699 (5.1\%) were being treated with a GLP1RA (73.8\% liraglutide, $23.5 \%$ exeOW, $2.7 \%$ lixisenatide). The numbers of patients who could be evaluated for CVOT eligibility were 124164 for EXSCEL, 116553 for PIONEER-6, 107040 for HARMONY, 106606 for LEADER, 105074 for REWIND and 98725 for SUSTAIN-6.

Patients being examined who were already taking a GLP-1RA at the visit were considered separately, because ongoing GLP-1RA therapy was an exclusion criterion in CVOTs. After applying I/E criteria (as outlined in Table S1), we calculated that the percentages of patients who were eligible for CVOTs were $35.8 \%$ for REWIND, $34.1 \%$ for PIONEER-6, $13.4 \%$ for EXSCEL, $10.1 \%$ for SUSTAIN-6, 9.5\% for HARMONY and $9.4 \%$ for LEADER. Overall, $45.4 \%$ of patients were eligible for at least one of the CVOTs being considered.

The clinical characteristics of patients treated with GLP-1RAs and of those who were eligible for CVOTs are provided in Table 1. 


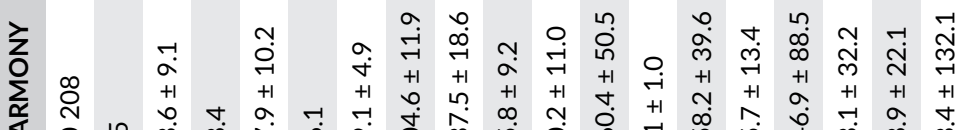

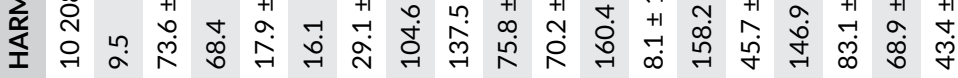

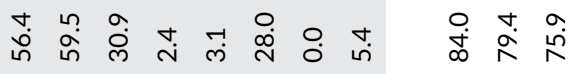

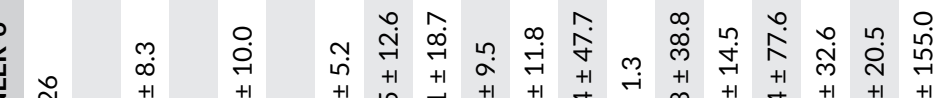

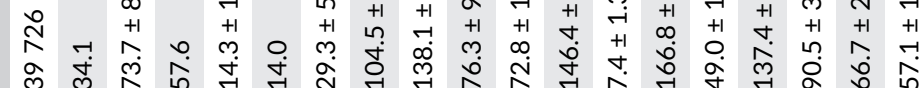

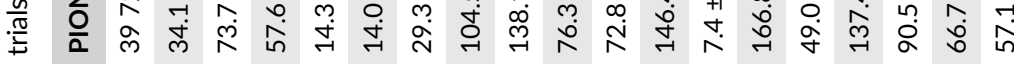

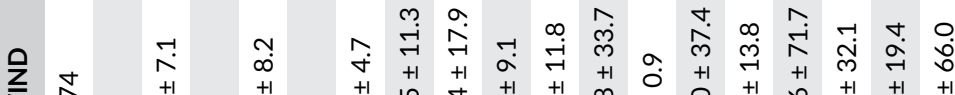

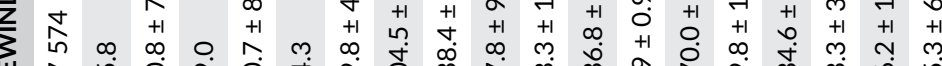

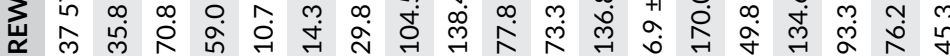

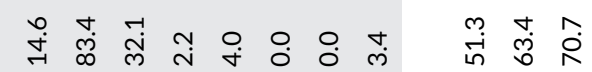

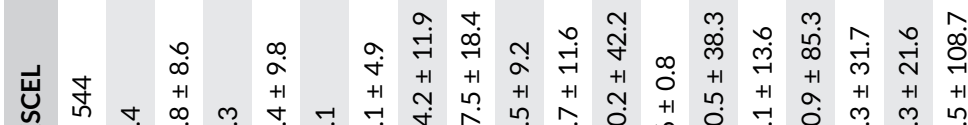

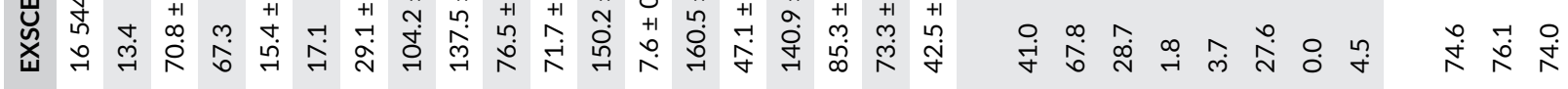

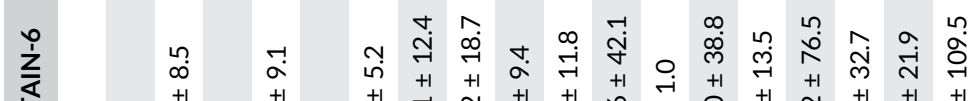

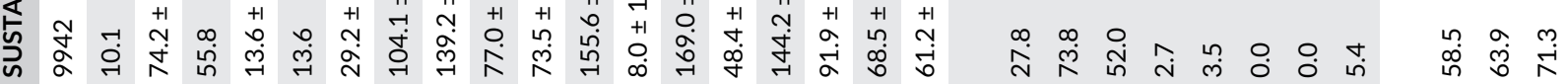

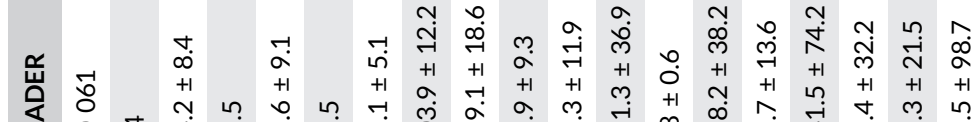
यु

岂

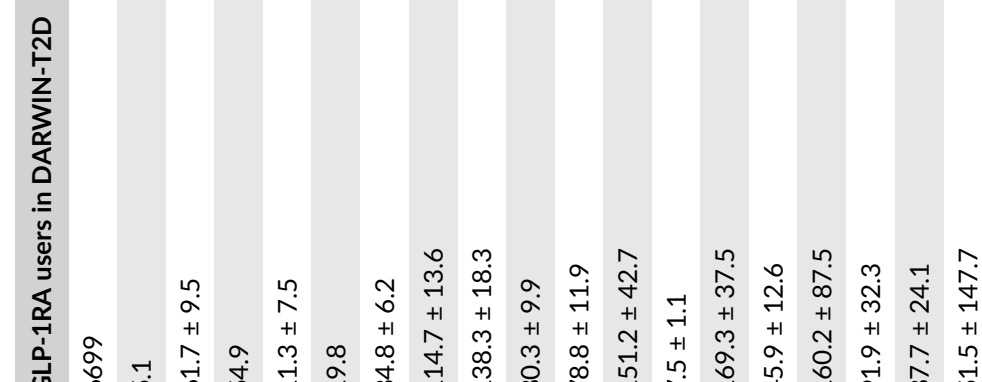

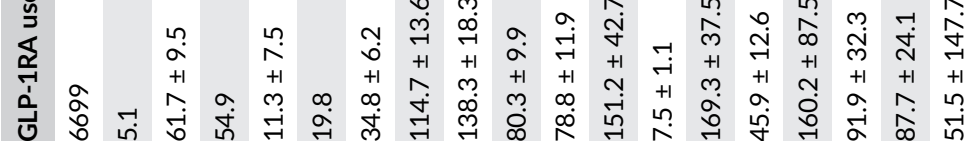

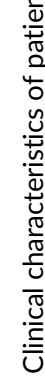

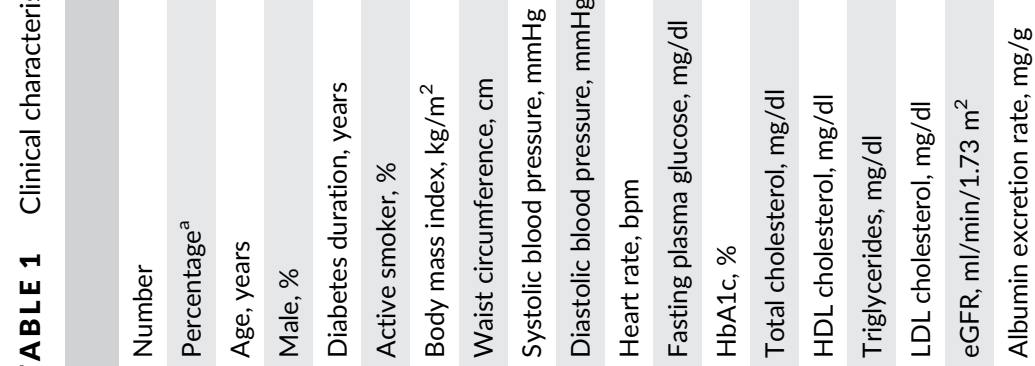

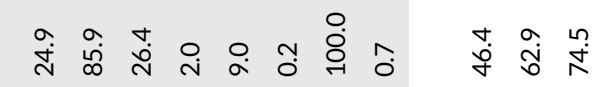




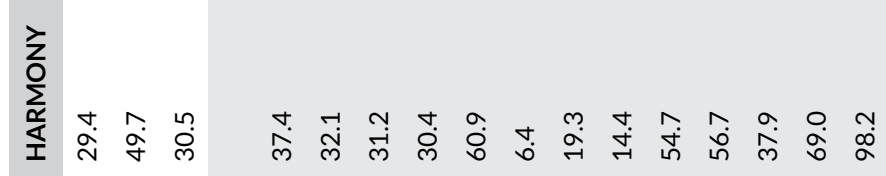

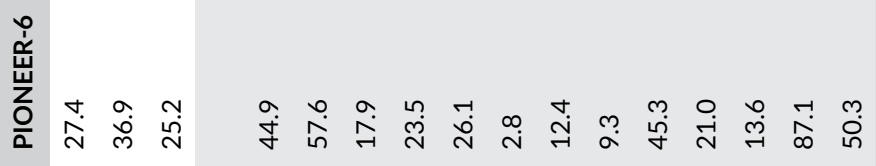

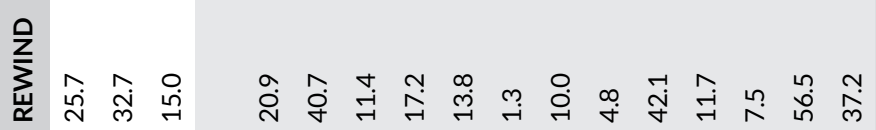

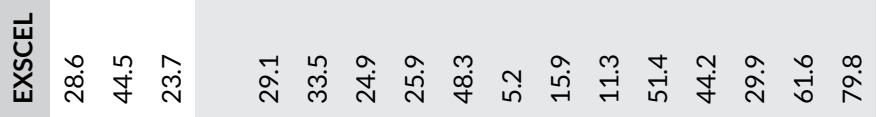

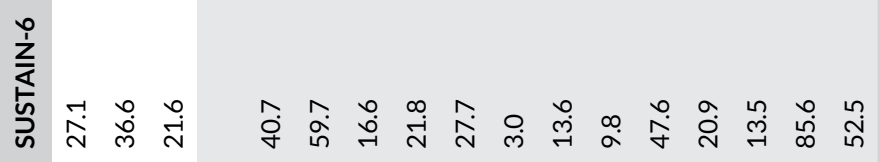

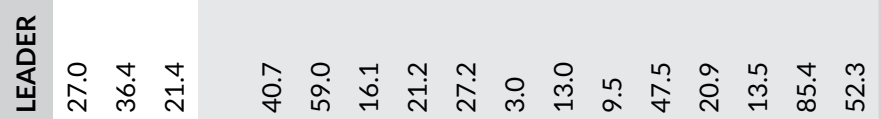

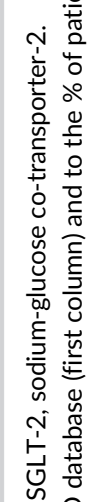

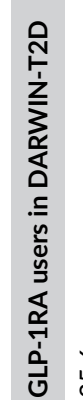

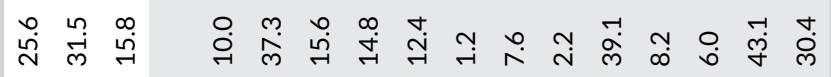

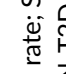

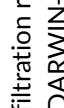

䓪

은 竞

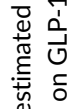

这

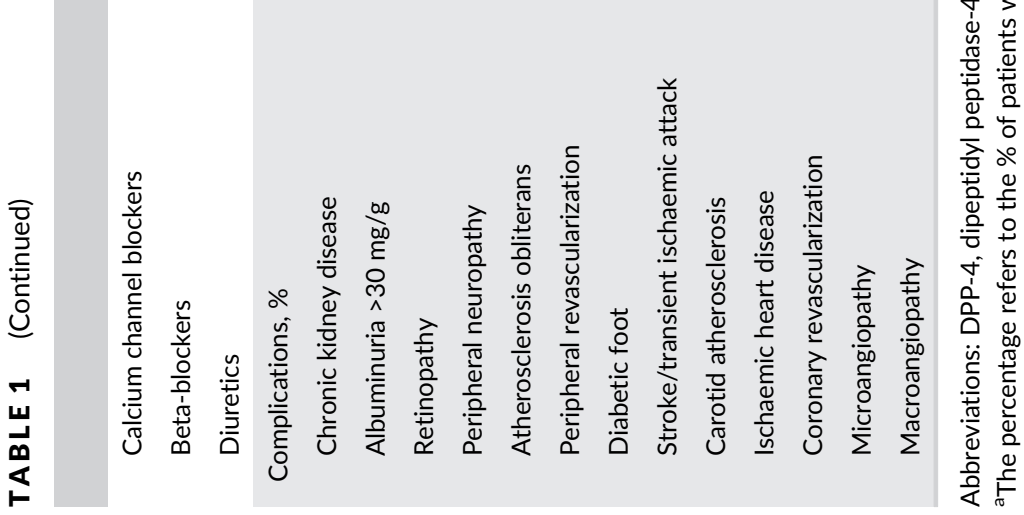



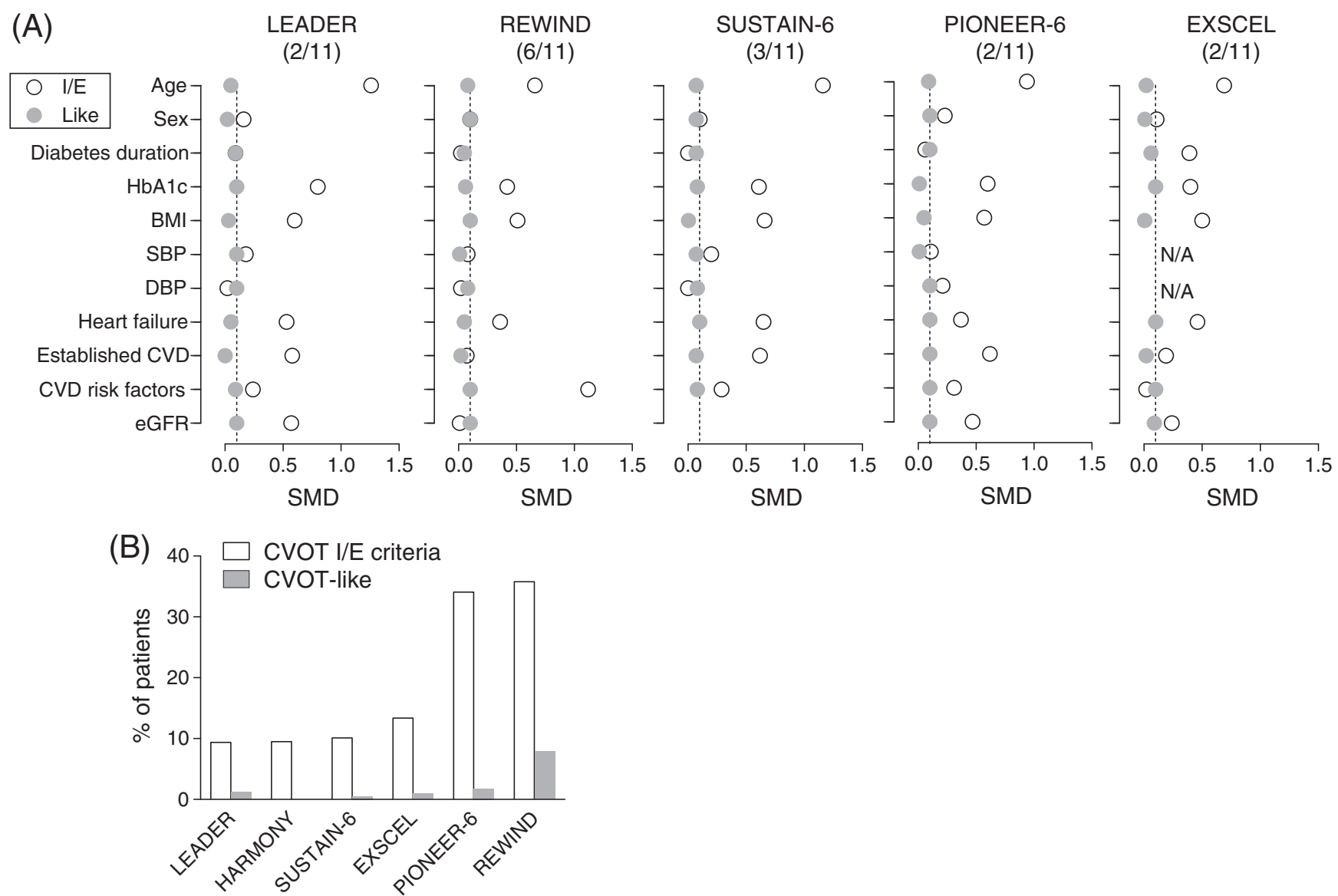

FIGURE 1 Real-world patients and cardiovascular outcome trials (CVOTs). (A) For each CVOT, the panels show the absolute standardized mean difference (SMD) between the actual trial population (retrieved from respective publications) and real-world patients selected based on inclusion/exclusion criteria (I/E) or for being CVOT-like (Like). In each plot, a dashed line indicates the SMD threshold of 0.1, indicating good balance. Fractions in brackets refer to the number of key clinical characteristics that are matched between real-world patients selected by I/E and trial characteristics. By design, all characteristics were balanced between CVOT-like patients and the respective CVOT population. (B) Proportion of real-world patients eligible for each CVOT based on I/E or sampled for being CVOT-like. BMI, body mass index; CVD, cardiovascular disease; DBP, diastolic blood pressure; eGFR, estimated glomerular filtration rate; N/A, not available; SBP, systolic blood pressure

We observed that the average clinical characteristics of patients who could be eligible for CVOTs were substantially different from the average clinical characteristics of patients who composed each CVOT population (Figure 1A). For instance, patients selected from the real-world database were older than in CVOTs. In addition, despite $80 \%-100 \%$ of patients in LEADER and SUSTAIN-6 having established cardiovascular disease, application of the I/E criteria to the real-world population yielded patients with a $70 \%-80 \%$ prevalence of microangiopathy (mostly chronic kidney disease) and a lower prevalence of macroangiopathy (40\%-50\%). Most other clinical characteristics were imbalanced between patients enrolled in CVOTs and real-world patients eligible for the same CVOTs (Table 2). Out of $11 \mathrm{key}$ clinical variables, eligible patients matched the trial characteristics with an absolute SMD of $<0.1$ for just two or three variables, with the notable exception of REWIND. Real-world patients eligible for REWIND were matched with the REWIND population for $6 / 11$ variables.
Patients who were already on a GLP-1RA showed substantially different clinical characteristics when compared with both those satisfying CVOT I/E criteria and those actually enrolled in CVOTs.

We then evaluated what proportion of real-world patients would constitute a population of individuals with key average characteristics similar to those enrolled in CVOTs. The largest datasets of real-world patients yielding CVOT-like populations were 0.5\% for SUSTAIN-6, 1.0\% for EXSCEL, $1.2 \%$ for LEADER, 1.8\% for PIONEER-6 and $7.9 \%$ for REWIND. We were unable to obtain a meaningful dataset of realworld patients who would match the population of the HARMONY study (Figure 1B).

\section{4 | DISCUSSION}

Although $10 \%-35 \%$ of real-world T2D patients could be enrolled in GLP-1RA CVOTs, their clinical characteristics were markedly different from those of CVOT populations. For the first time, we calculated that 
TAB LE 2 Key clinical characteristics of real-world patients compared with cardiovascular outcome trial (CVOT) patients. For each CVOT, we show the average clinical characteristics extracted from the respective publications, the characteristics of real-world patients who would be recruited into the CVOT based on inclusion/exclusion (I/E) criteria, and the characteristics of real-world patients sampled for being CVOT-like (Like). For both subgroups of real-world patients, we calculated the absolute standardized mean difference (SMD) as a measure of balance between groups; an SMD $\leq 0.10$ is conventionally considered indicative of a good balance

\begin{tabular}{|c|c|c|c|c|c|}
\hline Variable & LEADER & I/E & SMD & Like & SMD \\
\hline Number & 9340 & 10061 & & 1132 & \\
\hline Age, years & $64.3(7.2)$ & $74.2(8.4)$ & 1.26 & $64.6(7.6)$ & 0.05 \\
\hline Male, \% & 64.2 & 56.5 & 0.16 & 65.0 & 0.02 \\
\hline Diabetes duration, years & $12.8(8.0)$ & $13.6(9.1)$ & 0.09 & $13.5(8.4)$ & 0.09 \\
\hline $\mathrm{HbA} 1 \mathrm{c}, \%$ & $8.7(1.5)$ & $7.8(0.6)$ & 0.80 & $8.5(0.8)$ & 0.10 \\
\hline $\mathrm{BMI}, \mathrm{kg} / \mathrm{m}^{2}$ & $32.5(6.3)$ & $29.1(5.1)$ & 0.60 & $32.7(5.8)$ & 0.03 \\
\hline $\mathrm{SBP}, \mathrm{mmHg}$ & $135.9(17.7)$ & $139.1(18.6)$ & 0.18 & $137.8(18.7)$ & 0.10 \\
\hline $\mathrm{DBP}, \mathrm{mmHg}$ & $77.1(10.2)$ & $76.9(9.3)$ & 0.02 & $78.2(9.3)$ & 0.10 \\
\hline Heart failure, \% & 17.9 & 2.5 & 0.53 & 16.0 & 0.05 \\
\hline Established CVD, \% & 81.4 & 55.7 & 0.58 & 81.4 & 0.001 \\
\hline CVD risk factors, \% & 18.7 & 28.7 & 0.24 & 22.3 & 0.09 \\
\hline eGFR, $\mathrm{ml} / \mathrm{min} / 1.73 \mathrm{~m}^{2}$ & $80.4(21.0)$ & $68.3(21.5)$ & 0.57 & $78.0(26.5)$ & 0.10 \\
\hline Variable & REWIND & $\mathrm{I} / \mathrm{E}$ & SMD & Like & SMD \\
\hline Number & 9901 & 37574 & & 7280 & \\
\hline Age, years & $66.2(6.5)$ & $70.8(7.1)$ & 0.66 & $66.7(6.2)$ & 0.08 \\
\hline Male, \% & 53.9 & 59.0 & 0.10 & 59.3 & 0.10 \\
\hline Diabetes duration, years & $10.5(7.2)$ & $10.7(8.2)$ & 0.02 & $10.8(7.1)$ & 0.05 \\
\hline $\mathrm{HbA} 1 \mathrm{c}, \%$ & $7.3(1.1)$ & $6.9(0.9)$ & 0.42 & $7.4(1.2)$ & 0.06 \\
\hline $\mathrm{BMI}, \mathrm{kg} / \mathrm{m}^{2}$ & $32.3(5.7)$ & $29.8(4.7)$ & 0.51 & $31.9(5.3)$ & 0.10 \\
\hline $\mathrm{SBP}, \mathrm{mmHg}$ & $137.0(17.0)$ & $138.4(18.0)$ & 0.08 & $137.2(15.5)$ & 0.01 \\
\hline $\mathrm{DBP}, \mathrm{mmHg}$ & $78.0(9.9)$ & $77.8(9.1)$ & 0.02 & 78.7 (8.1) & 0.08 \\
\hline Heart failure, \% & 8.7 & 1.0 & 0.36 & 7.3 & 0.05 \\
\hline Established CVD, \% & 31.4 & 28.2 & 0.07 & 30.6 & 0.02 \\
\hline CVD risk factors, \% & 68.6 & 19.9 & 1.12 & 63.8 & 0.10 \\
\hline eGFR, $\mathrm{ml} / \mathrm{min} / 1.73 \mathrm{~m}^{2}$ & $75.0(22.1)$ & $75.2(21.2)$ & 0.009 & $77.4(22.2)$ & 0.10 \\
\hline Variable & SUSTAIN-6 & $\mathrm{I} / \mathrm{E}$ & SMD & Like & SMD \\
\hline Number & 3297 & 9942 & & 476 & \\
\hline Age, years & $64.6(7.4)$ & $74.2(8.5)$ & 1.16 & $65.1(6.7)$ & 0.07 \\
\hline Male, \% & 60.7 & 55.8 & 0.10 & 64.1 & 0.07 \\
\hline Diabetes duration, years & $13.9(8.1)$ & $13.6(9.1)$ & 0.00 & $14.5(6.8)$ & 0.07 \\
\hline $\mathrm{HbA} 1 \mathrm{c}, \%$ & $8.7(1.5)$ & $8.0(1.0)$ & 0.61 & $8.6(0.7)$ & 0.08 \\
\hline $\mathrm{BMI}, \mathrm{kg} / \mathrm{m}^{2}$ & $32.8(6.2)$ & $29.2(5.2)$ & 0.66 & $32.8(5.9)$ & 0.004 \\
\hline $\mathrm{SBP}, \mathrm{mmHg}$ & $135.6(17.2)$ & $139.2(18.7)$ & 0.20 & $136.8(17.5)$ & 0.07 \\
\hline $\mathrm{DBP}, \mathrm{mmHg}$ & $77.0(10.0)$ & $77.0(9.4)$ & 0.00 & $77.8(9.7)$ & 0.08 \\
\hline Heart failure, \% & 23.6 & 2.6 & 0.65 & 19.5 & 0.10 \\
\hline Established CVD, \% & 83.0 & 55.7 & 0.62 & 80.5 & 0.07 \\
\hline CVD risk factors, $\%$ & 17.0 & 29.0 & 0.29 & 14.1 & 0.08 \\
\hline eGFR, $\mathrm{ml} / \mathrm{min} / 1.73 \mathrm{~m}^{2}$ & N/A & N/A & N/A & N/A & N/A \\
\hline Variable & PIONEER-6 & $\mathrm{I} / \mathrm{E}$ & SMD & Like & SMD \\
\hline Number & 3183 & 39726 & & 1663 & \\
\hline Age, years & $66.0(7.0)$ & $73.7(8.3)$ & 0.94 & $66.6(7.2)$ & 0.09 \\
\hline Male, \% & 68.4 & 57.6 & 0.23 & 72.9 & 0.10 \\
\hline
\end{tabular}


TABLE 2 (Continued)

\begin{tabular}{|c|c|c|c|c|c|}
\hline Variable & LEADER & $\mathrm{I} / \mathrm{E}$ & SMD & Like & SMD \\
\hline Diabetes duration, years & $14.9(8.5)$ & $14.3(10.0)$ & 0.06 & $14.0(8.6)$ & 0.10 \\
\hline $\mathrm{HbA} 1 \mathrm{c}, \%$ & $8.2(1.6)$ & $7.4(1.3)$ & 0.60 & $8.2(0.7)$ & 0.01 \\
\hline $\mathrm{SBP}, \mathrm{mmHg}$ & $136.0(18.0)$ & $138.1(18.7)$ & 0.11 & $135.7(14.0)$ & 0.01 \\
\hline $\mathrm{DBP}, \mathrm{mmHg}$ & $74.0(21.0)$ & $76.3(9.5)$ & 0.21 & $77.0(8.0)$ & 0.10 \\
\hline Established CVD, \% & 84.7 & 58.1 & 0.62 & 80.7 & 0.10 \\
\hline CVD risk factors, $\%$ & 15.3 & 27.8 & 0.31 & 19.1 & 0.10 \\
\hline eGFR, $\mathrm{ml} / \mathrm{min} / 1.73 \mathrm{~m}^{2}$ & $76.0(10.0)$ & $66.2(21.3)$ & 0.47 & $71.6(26.0)$ & 0.10 \\
\hline Variable & EXSCEL & $\mathrm{I} / \mathrm{E}$ & SMD & Like & SMD \\
\hline Number & 14752 & 16544 & & 915 & \\
\hline $\mathrm{HbA} 1 \mathrm{c}, \%$ & $8.0(1.2)$ & $7.6(0.8)$ & 0.40 & $7.9(0.7)$ & 0.10 \\
\hline $\mathrm{BMI}, \mathrm{kg} / \mathrm{m}^{2}$ & $31.8(5.9)$ & $29.1(4.9)$ & 0.50 & $31.8(5.9)$ & 0.006 \\
\hline $\mathrm{SBP}, \mathrm{mmHg}$ & $\mathrm{N} / \mathrm{A}$ & $\mathrm{N} / \mathrm{A}$ & $\mathrm{N} / \mathrm{A}$ & $\mathrm{N} / \mathrm{A}$ & $\mathrm{N} / \mathrm{A}$ \\
\hline $\mathrm{DBP}, \mathrm{mmHg}$ & N/A & N/A & N/A & $\mathrm{N} / \mathrm{A}$ & N/A \\
\hline Heart failure, \% & 16.2 & 2.9 & 0.46 & 12.6 & 0.10 \\
\hline Established CVD, \% & 73.1 & 64.5 & 0.19 & 72.3 & 0.02 \\
\hline CVD risk factors, \% & 26.9 & 27.9 & 0.02 & 22.3 & 0.10 \\
\hline eGFR, $\mathrm{ml} / \mathrm{min} / 1.73 \mathrm{~m}^{2}$ & $76.3(22.9)$ & $70.5(25.5)$ & 0.24 & $78.4(31.5)$ & 0.09 \\
\hline $\mathrm{BMI}, \mathrm{kg} / \mathrm{m}^{2}$ & $32.3(5.9)$ & $29.1(4.9)$ & 0.59 & N/A & N/A \\
\hline $\mathrm{SBP}, \mathrm{mm} \mathrm{Hg}$ & $134.7(16.5)$ & $137.5(18.6)$ & 0.16 & $\mathrm{~N} / \mathrm{A}$ & N/A \\
\hline $\mathrm{DBP}, \mathrm{mm} \mathrm{Hg}$ & $76.8(10.1)$ & $75.8(9.2)$ & 0.10 & $\mathrm{~N} / \mathrm{A}$ & N/A \\
\hline Heart failure, \% & 20.0 & 4.3 & 0.50 & $\mathrm{~N} / \mathrm{A}$ & N/A \\
\hline Established CVD, \% & 100.0 & 85.4 & 0.58 & $\mathrm{~N} / \mathrm{A}$ & N/A \\
\hline CVD risk factors, $\%$ & 0.0 & 33.6 & 1.00 & $\mathrm{~N} / \mathrm{A}$ & N/A \\
\hline eGFR, $\mathrm{ml} / \mathrm{min} / 1.73 \mathrm{~m}^{2}$ & $79.0(25.5)$ & $68.9(22.1)$ & 0.42 & $\mathrm{~N} / \mathrm{A}$ & N/A \\
\hline
\end{tabular}

Abbreviations: BMI, body mass index; CVD, cardiovascular disease; DBP, diastolic blood pressure; eGFR, estimated glomerular filtration rate; N/A, not available; SBP, systolic blood pressure.

Established CVD and CVD risk factors are defined as described in each trial publication and slightly modified (as illustrated in Table S1).

the proportion of real-world patients who have true CVOT-like characteristics is much smaller, ranging from $0.5 \%$ to $7.9 \%$.

CVOTs have shown the notable capacity of some GLP-1RAs to reduce the rate of adverse cardiovascular outcomes in patients with T2D. ${ }^{9}$ The generalizability of such findings to clinical practice is challenging, in particular because the populations investigated in CVOTs are markedly different from real-world T2D patients. CVOTs select patients whose characteristics are intended to maximize the probability of trial success, whereas the representativeness of the real-world T2D population is rarely an issue considered in CVOT design.

Prior studies have examined what proportion of patients from clinical practice databases would be eligible for CVOTs on GLP-1RAs or SGLT-2is. By analysing US adult T2D databases, Boye et al ${ }^{13}$ reported proportions of patients eligible for LEADER, SUSTAIN-6, EXSCEL and REWIND that were quite similar to those shown in our 
study. This comparison is important because the proportion of patients eligible for CVOTs would be expected to be higher in US than in European databases, since CVOTs were conducted in the US. While small differences were probably a result of regional variations (eg, in $\mathrm{BMI}$ ), no major difference emerged, suggesting a limited impact of geographical and cultural factors.

Similar analyses have been performed on CVOTs for SGLT-2is. ${ }^{12,14}$ Nicolucci et al ${ }^{11}$ showed that real-world T2D patients eligible for CVOTs on SGLT-2is were different to trial populations in many instances. ${ }^{11}$ To date, however, no study has been able to calculate what proportion of real-world patients truly constitute CVOT-like populations.

By analysing GLP-1RA CVOTs, we found substantial differences between the eligible real-world populations and trial populations. The high proportion of patients eligible for PIONEER- $6^{8}$ in our study reflects enrolment criteria that, different to those of EXSCEL, ${ }^{7}$ lacked constraint on the ratio between patients with established cardiovascular disease and those with multiple cardiovascular risk factors. However, the resulting PIONEER-6 eligible subset was greatly imbalanced compared with the true PIONEER- 6 population. ${ }^{8}$ We therefore examined what proportion of patients from the real-world database would generate CVOT-like populations. To this end, we used a Bayesian method to sample patients from a large dataset based on given average clinical characteristics. We found that the greatest subset of patients with CVOT-like characteristics was much smaller than the proportion of eligible patients. Interestingly, we found no subset of real-world patients matching the HARMONY trial population, probably because HARMONY patients had established cardiovascular disease at a young age. ${ }^{4}$ Notably, REWIND was confirmed as the CVOT mostly represented within the T2D population, although only $7.9 \%$ of real-world patients were truly REWIND-like. On the contrary, the apparently large generalizability of PIONEER-6 based on I/E criteria was not confirmed. This important finding highlights that CVOT populations are extremely specific and that they are poorly represented by real-world T2D patients.

We acknowledge that, in view of the potentially wide cardiovascular benefits of GLP-1RAs, this class of GLMs is substantially underutilized among T2D patients. ${ }^{22}$ However, our data argue that the generalizability of trial populations to clinical practice should not be based on trial I/E criteria. It is important to remember that the representativeness of trial populations is only one aspect of trial-finding generalizability. Observational studies, although unable to substitute for CVOTs, ${ }^{23}$ complement CVOT findings and can provide an estimate of true effectiveness in real-world patients often not represented in CVOTs. Real-world studies on the glycaemic and extra-glycaemic effectiveness of GLP-1RAs have confirmed findings from phase III $\mathrm{RCTs},{ }^{24-26}$ but there is still a paucity of cardiovascular real-world studies. ${ }^{27}$ In a small study from the UK, the intensification of oral therapy by adding GLP-1RAs was associated with a lower cardiovascular events rate than the intensification with insulin. ${ }^{28}$ A study using the Swedish and Danish diabetes registries reported that liraglutide, compared with dipeptidyl peptidase-4 inhibitors, only exerted significant cardiovascular protection in patients with established cardiovascular disease. ${ }^{29}$
In parallel to observational studies, alternative methods have recently been developed to transport trial findings to target populations using an inverse odds weighting approach ${ }^{30,31}$.

Our study has limitations inherent to the nature of the data being analysed. Real-world databases often lack some of the information required to address trial I/E criteria, leading to the need for simplifications (Table S1). Exclusion of patients with missing data can lead to deviations from the target population, potentially affecting generalizability analysis. ${ }^{32}$ Under-reporting may be another issue in the data collected for clinical purposes, possibly leading to an underestimation of the proportion of patients eligible for CVOTs. Finally, we excluded prevalent GLP-1RA users from the analysis of generalizability because it was expected that GLP-1RAs would have already determined at least part of their benefits in those patients. However, the clinical characteristics of the small group of patients on GLP-1RAs did not resemble those of CVOT populations, suggesting that excluding prevalent GLP-1RA users was unlikely to affect the generalizability findings in a meaningful manner.

In conclusion, we show that applying CVOT enrolment criteria to real-world populations leads to a huge overestimation of patients who resemble CVOT populations, thereby leaving open the issue of generalizability.

\section{ACKNOWLEDGMENTS}

We wish to thank Alessia Russo, secretariat of the Italian Diabetes Society, for the invaluable technical support.

This study was supported by the Italian Diabetes Society and the University of Padova.

\section{CONFLICT OF INTEREST}

G.P.F. received grant support, lecture or advisory board fees from AstraZeneca, Boehringer-Ingelheim, Eli Lilly, Mundipharma, NovoNordisk, Sanofi, Genzyme, Servier, Abbott, Novartis and Merck Sharp \& Dohme. E.O. received lecture fees from Eli Lilly and Novo Nordisk. O.L. has received speaker fees from Eli-Lilly and NovoNordisk; she also received research grants from AstraZeneca and advisory board fees from NovoNordisk. S.M. received lecture or advisory board fees from AstraZeneca, Sanofi and Takeda. A.C. has received consultancy or speaker fees from Abbott, AstraZeneca, Boehringer-Ingelheim, Bruno Farmaceutici, Janssen, Eli-Lilly, Merck Sharp \& Dohme, Novartis, NovoNordisk, Roche, Sanofi-Aventis, Servier and Takeda; he also received research grants From Eli-Lilly and NovoNordisk. A.A. received research grants, lecture or advisory board fees from Merck Sharp \& Dome, AstraZeneca, Novartis, Boeringher-Ingelheim, Sanofi, Mediolanum, Janssen and NovoNordisk. V.S., P.B. and F.Q. have nothing to disclose.

\section{AUTHOR CONTRIBUTIONS}

Study design: V.S., P.B., A.C., A.A. and G.P.F. Data collection and analysis: V.S., P.B., E.O., O.L., S.M., F.Q. and G.P.F. Manuscript writing: V.S., P.B., A.C., A.A. and G.P.F. Manuscript revision: E.O., O.L., S.M. and F.Q. All of the authors approved the final version of the manuscript. 


\section{COMPOSITION OF THE DARWIN-T2D DATABASE}

Agostino Consoli and Gloria Formoso (Dipartimento di Medicina e Scienze dell'Invecchiamento - Università Degli studi G. D'Annunzio di Chieti-Pescara); Giovanni Grossi (Ospedale San Francesco di Paola Azienda Sanitaria Provinciale di Cosenza); Achiropita Pucci (Azienda Sanitaria Provinciale di Cosenza); Giorgio Sesti and Francesco Andreozzi (Azienda Ospedaliero Universitaria di Catanzaro); Giuseppe Capobianco (Azienda Sanitaria Locale Napoli 2 Nord); Adriano Gatti (Ospedale San Gennaro dei Poveri - Azienda Sanitaria Locale Napoli 1 Centro); Riccardo Bonadonna, Ivana Zavaroni and Alessandra Dei Cas (Azienda Ospedaliero Universitaria di Parma); Giuseppe Felace (Ospedale di Spilimbergo - Azienda per l'Assistenza Sanitaria n.5 Friuli Occidentale); Patrizia Li Volsi (Ospedale di Pordenone - Azienda per l'Assistenza Sanitaria n.5 Friuli Occidentale); Raffaella Buzzetti and Gaetano Leto (Ospedale Santa Maria Goretti - Azienda Sanitaria Locale di Latina); Gian Pio Sorice (Fondazione Policlinico Universitario A. Gemelli, Roma); Paola D'Angelo (Ospedale Sandro Pertini - Azienda Sanitaria Locale Roma 2); Susanna Morano (Azienda Ospedaliera Universitaria Policlinico Umberto I, Roma); Antonio Carlo Bossi (Ospedale di Treviglio - Azienda Socio Sanitaria Territoriale Bergamo Ovest); Edoardo Duratorre (Ospedale Luini Confalonieri di Luino - Azienda Socio Sanitaria Territoriale Sette Laghi); Ivano Franzetti (Ospedale Sant'Antonio Abate di Gallarate - Azienda Socio Sanitaria Territoriale Valle Olona); Paola Silvia Morpurgo (Ospedale Fatebenefratelli Azienda Socio Sanitaria Territoriale Fatebenefratelli Sacco); Emanuela Orsi (Fondazione IRCCS Ca' Granda - Ospedale Maggiore Policlinico di Milano); Fabrizio Querci (Ospedale Pesenti Fenaroli di Alzano Lombardo - Azienda Socio Sanitaria Territoriale Bergamo Est); Massimo Boemi† and Federica D'Angelo (Presidio Ospedaliero di Ricerca INRCA-IRCCS di Ancona); Massimiliano Petrelli (Azienda Ospedaliero Universitaria Ospedali Riuniti di Ancona); Gianluca Aimaretti and loannis Karamouzis (Azienda Ospedaliero Universitaria Maggiore della Carità di Novara); Franco Cavalot (Azienda Ospedaliero Universitaria San Luigi Gonzaga, Orbassano); Giuseppe Saglietti† (Ospedale Madonna del Popolo di Omegna - Azienda Sanitaria Locale Verbano Cusio Ossola); Giuliana Cazzetta (Casa della Salute, Ugento - Distretto Socio Sanitario Gagliano del Capo - Azienda Sanitaria Locale di Lecce); Silvestre Cervone (Presidio ospedaliero San Marco in Lamis - Distretto Socio Sanitario San Marco in Lamis - Azienda Sanitaria Locale di Foggia); Eleonora Devangelio (Distretto Socio Sanitario di Massafra Azienda Sanitaria Locale di Taranto); Olga Lamacchia (Azienda Ospedaliero Universitaria Ospedali Riuniti di Foggia); Salvatore Arena (Ospedale Umberto I - Azienda Sanitaria Provinciale di Siracusa); Antonino Di Benedetto (Azienda Ospedaliera Universitaria Policlinico G. Martino di Messina); Lucia Frittitta (Azienda Ospedaliera di Rilievo Nazionale e di Alta Specializzazione Garibaldi di Catania); Carla Giordano (Azienda Universitaria Policlinico Paolo Giaccone di Palermo); Salvatore Piro (Azienda Ospedaliera di Rilievo Nazionale e di Alta Specializzazione Garibaldi di Catania); Manfredi Rizzo, Roberta Chianetta and Carlo Mannina (Azienda Universitaria Policlinico Paolo Giaccone di Palermo); Roberto Anichini (Ospedale San Jacopo di Pistoia - Azienda USL Toscana Centro); Giuseppe Penno (Azienda Ospedaliero Universitaria Pisana); Anna Solini (Azienda Ospedaliera
Universitaria Pisana); Bruno Fattor (Comprensorio Sanitario di Bolzano - Azienda Sanitaria della Provincia Autonoma di Bolzano); Enzo Bonora and Massimo Cigolini (Azienda Ospedaliero Universitaria Integrata di Verona); Annunziata Lapolla and Nino Cristiano Chilelli (Complesso Socio Sanitario Ai Colli - Azienda ULSS n.6 Euganea); Maurizio Poli (Ospedale Girolamo Fracastoro di San Bonifacio Azienda ULSS n.9 Scaligera); Natalino Simioni and Vera Frison (Ospedale di Cittadella - Azienda ULSS n.6 Euganea); Carmela Vinci (Azienda ULSS n.4 Veneto Orientale).

\section{ORCID}

Angelo Avogaro (D) https://orcid.org/0000-0002-1177-0516 Gian Paolo Fadini (iD https://orcid.org/0000-0002-6510-2097

\section{REFERENCES}

1. Kieffer CM, Robertson AS. Impact of FDA-required cardiovascular outcome trials on type 2 diabetes clinical study initiation from 2008 to 2017. Ther Innov Regul Sci. 2019;2168479019860122.

2. Davies MJ, D'Alessio DA, Fradkin J, et al. Management of hyperglycaemia in type 2 diabetes, 2018. A consensus report by the American Diabetes Association (ADA) and the European Association for the Study of Diabetes (EASD). Diabetologia. 2018;61:2461-2498.

3. Gerstein HC, Colhoun HM, Dagenais GR, et al. Dulaglutide and cardiovascular outcomes in type 2 diabetes (REWIND): a double-blind, randomised placebo-controlled trial. Lancet. 2019;394:121-130.

4. Hernandez AF, Green JB, Janmohamed S, et al. Albiglutide and cardiovascular outcomes in patients with type 2 diabetes and cardiovascular disease (Harmony Outcomes): a double-blind, randomised placebocontrolled trial. Lancet. 2018;392:1519-1529.

5. Marso SP, Bain SC, Consoli A, et al. Semaglutide and cardiovascular outcomes in patients with type 2 diabetes. N Engl J Med. 2016;375: 1834-1844.

6. Marso SP, Daniels GH, Brown-Frandsen K, et al. Liraglutide and cardiovascular outcomes in type 2 diabetes. N Engl J Med. 2016;375: 311-322.

7. Holman RR, Bethel MA, Mentz RJ, et al. Effects of once-weekly exenatide on cardiovascular outcomes in type 2 diabetes. $N$ Engl J Med. 2017;377:1228-1239.

8. Husain M, Birkenfeld AL, Donsmark $M$, et al. Oral semaglutide and cardiovascular outcomes in patients with type 2 diabetes. $N$ Engl J Med. 2019;381:841-851.

9. Kristensen SL, Rorth R, Jhund PS, et al. Cardiovascular, mortality, and kidney outcomes with GLP-1 receptor agonists in patients with type 2 diabetes: a systematic review and meta-analysis of cardiovascular outcome trials. Lancet Diabetes Endocrinol. 2019;7:776-785.

10. Mannucci E, Dicembrini I, Nreu B, Monami M. Glucagon-like peptide1 receptor agonists and cardiovascular outcomes in patients with and without prior cardiovascular events: an updated meta-analysis and subgroup analysis of randomized controlled trials. Diabetes Obes Metab. 2020;22:203-211.

11. Nicolucci A, Candido R, Cucinotta D, et al. Generalizability of cardiovascular safety trials on SGLT2 inhibitors to the real world: implications for clinical practice. Adv Ther. 2019;36:2895-2909.

12. Birkeland KI, Bodegard J, Norhammar A, et al. How representative of a general type 2 diabetes population are patients included in cardiovascular outcome trials with SGLT2 inhibitors?. Diabetes Obes Metab: A large European observational study; 2018. [Epub ahead of print].

13. Boye KS, Riddle MC, Gerstein HC, et al. Generalizability of glucagonlike peptide-1 receptor agonist cardiovascular outcome trials to the overall type 2 diabetes population in the United States. Diabetes Obes Metab. 2019;21:1299-1304. 
14. Wittbrodt E, Chamberlain D, Arnold SV, Tang F, Kosiborod M. Eligibility of patients with type 2 diabetes for sodium-glucose co-transporter-2 inhibitor cardiovascular outcomes trials: An assessment using the Diabetes Collaborative Registry. Diabetes Obes Metab. 2019;21:1985-1989.

15. Fadini GP, Zatti G, Consoli A, Bonora E, Sesti G, Avogaro A. Rationale and design of the DARWIN-T2D (DApagliflozin Real World evldeNce in Type 2 Diabetes): A multicenter retrospective nationwide Italian study and crowdsourcing opportunity. Nutr Metab Cardiovasc Dis. 2017;27:1089-1097.

16. Fadini GP, Zatti G, Baldi I, et al. Use and effectiveness of dapagliflozin in routine clinical practice: An Italian multicentre retrospective study. Diabetes Obes Metab. 2018;20:1781-1786.

17. Levey AS, Stevens LA, Schmid $\mathrm{CH}$, et al. A new equation to estimate glomerular filtration rate. Ann Intern Med. 2009;150:604-612.

18. Ceriello A, Rossi MC, De Cosmo S, et al. Overall quality of care predicts the variability of key risk factors for complications in type 2 diabetes: an observational, longitudinal retrospective study. Diabetes Care. 2019;42:514-519.

19. Colombo D, Maathuis MH. Order-independent constraint-based causal structure learning. J Mach Learn Res. 2014;15:3921-3962.

20. Broom BM, Do KA, Subramanian D. Model averaging strategies for structure learning in Bayesian networks with limited data. BMC Bioinformatics. 2012;13:S10.

21. Lauritzen SL. Graphical Models. Oxford Statistical Science Series 1996. Oxford, UK: Clarendon Press.

22. Fadini GP, Frison V, Rigato M, et al. Trend 2010-2018 in the clinical use of GLP-1 receptor agonists for the treatment of type 2 diabetes in routine clinical practice: an observational study from Northeast Italy. Acta Diabetol. 2019. [Epub ahead of print].

23. Gerstein HC, McMurray J, Holman RR. Real-world studies no substitute for RCTs in establishing efficacy. Lancet. 2019;393:210-211.

24. Morieri ML, Rigato M, Frison V, et al. Fixed versus flexible combination of GLP-1 receptor agonists with basal insulin in type 2 diabetes: A retrospective multicentre comparative effectiveness study. Diabetes Obes Metab. 2019;21:2542-2552.

25. Fadini GP, Sciannameo V, Franzetti I, et al. Similar effectiveness of dapagliflozin and GLP-1 receptor agonists concerning combined endpoints in routine clinical practice: A multicentre retrospective study. Diabetes Obes Metab. 2019;21:1886-1894.
26. Mody R, Huang Q, Yu M, et al. Adherence, persistence, glycaemic control and costs among patients with type 2 diabetes initiating dulaglutide compared with liraglutide or exenatide once weekly at 12-month follow-up in a real-world setting in the United States. Diabetes Obes Metab. 2018. [Epub ahead of print].

27. Chatterjee S, Davies MJ, Khunti K. What have we learnt from "real world" data, observational studies and meta-analyses. Diabetes Obes Metab. 2018;20(Suppl 1):47-58.

28. Anyanwagu U, Mamza J, Mehta R, Donnelly R, Idris I. Cardiovascular events and all-cause mortality with insulin versus glucagonlike peptide-1 analogue in type 2 diabetes. Heart. 2016;102: 1581-1587.

29. Svanstrom H, Ueda P, Melbye M, et al. Use of liraglutide and risk of major cardiovascular events: a register-based cohort study in Denmark and Sweden. Lancet Diabetes Endocrinol. 2019;7:106-114.

30. Hong JL, Webster-Clark M, Jonsson Funk M, et al. Comparison of methods to generalize randomized clinical trial results without individual-level data for the target population. Am J Epidemiol. 2019; 188:426-437.

31. Westreich D, Edwards JK, Lesko CR, Stuart E, Cole SR. Transportability of trial results using inverse odds of sampling weights. Am J Epidemiol. 2017;186:1010-1014.

32. Hong JL, Jonsson Funk M, LoCasale R, et al. Generalizing randomized clinical trial results: implementation and challenges related to missing data in the target population. Am J Epidemiol. 2018;187:817-827.

\section{SUPPORTING INFORMATION}

Additional supporting information may be found online in the Supporting Information section at the end of this article.

How to cite this article: Sciannameo V, Berchialla P, Orsi E, et al. Enrolment criteria for diabetes cardiovascular outcome trials do not inform on generalizability to clinical practice: The case of glucagon-like peptide-1 receptor agonists. Diabetes Obes Metab. 2020;22:817-827. https://doi.org/10.1111/dom. 13962 